\title{
Mobile Vision-based Automatic Counting of Bacteria Colonies
}

\author{
Jacey-Lynn Minoi, Tin Tze Chiang, Terrin \\ Lim \\ Faculty of Computer Science and \\ Information Technology, \\ Universiti Malaysia Sarawak \\ 94300 Kota Samarahan, Sarawak Malaysia \\ Email: jaceylynnminoi@gmail.com
}

\author{
Zaharin Yusoff \\ Computer Science Department \\ Faculty of Defence Science and Technology \\ National Defence University of Malaysia \\ Sungai Besi Camp 57000 Kuala Lumpur \\ Malaysia \\ Email: zarinby@gmail.com
}

\author{
Abdul Hafiz Abdul Karim, Azham \\ Zulharnain \\ Faculty of Engineering, \\ Faculty of Resource Science and \\ Technology, \\ Universiti Malaysia Sarawak \\ 94300 Kota Samarahan, Sarawak \\ Malaysia
}

\begin{abstract}
The procedure for counting colonies is often performed manually and the process is lengthy and tedious. For that reason, several methods that rely on digital images for automatically counting cells and bacteria colonies have been proposed. Fully automated and high throughput hardware imaging instruments are also available, but such machines are extremely costly. In this paper, we introduce a mobile based computer vision algorithm for automatic bacteria colony counting using morphological operations and transforms in image processing, on a custom Android mobile cross-platform open source software and written in Java, $\mathrm{C}++$ and Open CV computer vision library. The results have shown are promising given that the acquisition and detection were done in a noncontrolled environment.
\end{abstract}

Keywords - Colony counting, Hough transform, wavelet

\section{INTRODUCTION}

Determining the number of bacteria colonies, such as E.Coli, S. Cerevisiae yeast, is a standard procedure in microbiological experiments. The major issue in the laboratory is to produce a reliable estimation for the number of bacteria colonies in Petri plates. The counting process is still primarily done manually using a marker to mark each spot on the plate so as not to double count, and it works fine for a small number of plates. However, a microbiologist in a laboratory usually deals with at least 200 Petri plates in a day, and thus this manual counting task can be laborious, daunting, time consuming and also inaccurate, either due to biasedness or errors induced by eye fatigue. Some researchers have adopted advanced automatic imaging counting systems, but these instruments are extremely expensive to be implemented in small laboratories that perform counting only occasionally. On the other hand, larger laboratories with tremendously large counting needs would require a few of such automatic counter systems.

Various studies have proposed imaging techniques for automatic counting of certain types of objects, such as cells, bacteria, people, tree crowns, etc., as their main components [1]. Reliable extraction of centroids from bacteria cells is an important task to ensure that the cells are properly segmented, quantified and recognised. The simplest approach in colony counting is to correlate the contour of the colonies with a circular pattern based on the local maxima in the correlation map [2]. Another approach exploits a particular form of lighting by injecting fluorogenic substrates to produce fluorescence images [3]. Given the high contrast in the produced images, colonies can be easily detected. However, this approach may not work well if the contrast in the fluorescence images is low. Some apply the Watershed transform method to split and segment adjacent colonies $[4,5]$. Another popular method is the Hough transform, also to split touching colonies but it may tend to incorrectly detect adjacent colonies as a single colony object [6]. There is also the morphological analysis approach for counting, as introduced in [7], based on the fact that bacteria colony types come in different forms - circular, irregular, filamentous and rhizoid. These methods usually rely on manual hand-tuning the threshold values in a controlled environment. Other more advanced methods include the spectroscopic technique [8], light scattering approach [9], fibre optic technology [10], and flow cytometry [11]. However, the accurate segmentation of bacteria remains an issue to be resolved, especially due to the different types of bacteria that produce different shape colonies, and for cases of colonies being very close or adjacent to one another.

Existing image processing tools to count bacteria colonies are essentially software-centred computer systems, where images are captured using digital cameras. These software are either proprietary to the research lab or sold at a very high cost. Given the increase of smart mobile devices among users, it is our aim to develop a mobile based computer vision technique to automate the process of detection and counting of colonies to assists microbiologists to tract the colony growth over a time series.

With the recent significant increase of mobile devices such as smartphones and tablets, the expected release of mobile apps for the benefit of laboratory research is also rising. Within these couple of years, a broad portfolio of scientific mobile apps for both iOS and Android devices has been developed and these would transform today's biology 